\title{
On Dual-Hyperbolic Numbers with Generalized Fibonacci and Lucas Numbers Components
}

\author{
Mehmet Ali Güngör ${ }^{1 *}$ and Arzu Cihan ${ }^{1}$ \\ ${ }^{1}$ Department of Mathematics, Faculty of Science and Arts, Sakarya University, Sakarya, Turkey \\ *Corresponding author E-mail: agungor@sakarya.edu.tr
}

\section{Article Info}

Keywords: Dual-hyperbolic numbers, Generalized Fibonacci numbers, Generalized Lucas numbers

2010 AMS: 11B39, $11 B 83$

Received: 09 September 2019

Accepted: 24 October 2019

Available online: 20 December 2019

\begin{abstract}
Dual-hyperbolic Fibonacci and Lucas numbers with Fibonacci and Lucas coefficients are introduced by Cihan et al. and some identities and theorems are given regarding modules and conjugates of these numbers. Later, generating function and Binet's formula with the help of this generating function have been derived. Also, Binet formula, Cassini's, Catalan's, d'Ocagne's, Honsberger and Tagiuri identities are found for dual-hyperbolic numbers with generalized Fibonacci and Lucas coefficients. While these operations are being done, we will benefit from the well-known Fibonacci and Lucas identitites. Moreover, it is seen that the results which are obtained for the values $p=1$ and $q=0$ corresponds to the theorems in the article by Cihan et al. [1].
\end{abstract}

\section{Introduction}

Italian mathematician Leonardo Fibonacci's Liber Abaci was one of the most important books on mathematics in the Middle Ages. Through this book mathematicians introduced Fibonacci number sequence concept. Several studies have been conducted with respect to Fibonacci numbers and Fibonacci quaternions [2]-[5].

Dual-hyperbolic numbers with Fibonacci and Lucas coefficients which is constitutes a new number system have been introduced by Cihan and her colleagues [1]. In this article, the dual-hyperbolic number system has been generalized based on the article [1].

Firstly, addition, multiplication, modules and conjugates of these numbers have been defined and the fundamental identities for these numbers regarding these operations have been proven. Then, we have defined generating function and this function helped us to find Binet's formula. Additionally, d'Ocagne's, Honsberger, Tagiuri, Catalan identities have been obtained and Cassini's identity has been given in case of $r=1$ for the Catalan identity. Finally, we have discussed special cases and have given examples.

\section{Preliminaries}

The Fibonacci and Lucas numbers have many interesting properties and applications. Initial conditions for the Fibonacci and Lucas numbers are defined as follows respectively

$$
F_{0}=0, \quad F_{1}=1, \ldots, \quad F_{n+1}=F_{n}+F_{n-1}, \quad n \geq 1
$$

and

$$
L_{0}=2, \quad L_{1}=1, \ldots, \quad L_{n+1}=L_{n}+L_{n-1}, \quad n \geq 1
$$

where $F_{n}$ and $L_{n}$ denote the $n$-th Fibonacci and Lucas numbers, respectively.

Binet formula for the $n$-th Fibonacci and Lucas numbers are given by the following relation

$$
F_{n}=\frac{1}{\sqrt{5}}\left(\alpha^{n}-\beta^{n}\right), \quad L_{n}=\alpha^{n}+\beta^{n}, \quad n \geq 1
$$

Email addresses and ORCID numbers: agungor@sakarya.edu.tr, https://orcid.org/0000-0003-1863-3183 (M. A. Güngör), arzu.cihan3@ogr.sakarya.edu.tr, https://orcid.org/0000-0003-2003-3507 (A. Cihan) 
(see [2]).

On the other hand, Horadam introduced generalized Fibonacci numbers with the initial conditions as follows

$$
H_{1}=p, \quad H_{2}=p+q, \quad p, q \in Z
$$

where the recurrence relation is defined by

$$
H_{n}=H_{n-1}+H_{n-2}, \quad n \geq 3
$$

or

$$
H_{n}=(p-q) F_{n}+q F_{n+1}
$$

In the above equation, if we take $p=1$ and $q=0$, then the generalized Fibonacci number becomes Fibonacci number. If we take $p=1$ and $q=2$, then the generalized Lucas number becomes Lucas number. Furthermore, Horadam investigated Binet formula for the $n$-th generalized Fibonacci number such that

$$
H_{n}=\frac{1}{2 \sqrt{5}}\left(\alpha^{n}-\mu \beta^{n}\right)
$$

(see [2]).

The set of dual-hyperbolic numbers is defined by

$$
D H=\left\{w=z_{1}+z_{2} \varepsilon \mid z_{1}, z_{2} \in \text { Hwere, } \varepsilon^{2}=0, \varepsilon \neq 0\right\} .
$$

If we consider two hyperbolic numbers $z_{1}=x_{1}+x_{2} j$ and $z_{2}=y_{1}+y_{2} j$, then any dual-hyperbolic number can be written as

$$
w=x_{1}+x_{2} j+y_{1} \varepsilon+y_{2} j \varepsilon .
$$

There exist five different conjugates and these conjugates are given as follows

$$
\begin{aligned}
& |\omega|^{\dagger_{1}}=\bar{z}_{1}+\varepsilon \bar{z}_{2}, \quad \text { hyperbolic conjugation } \\
& |\omega|^{\dagger_{2}}=z_{1}-\varepsilon z_{2}, \quad \text { dual conjugation } \\
& |\omega|^{\dagger_{3}}=\bar{z}_{1}-\varepsilon \bar{z}_{2}, \quad \text { coupled conjugation } \\
& |\omega|^{\dagger_{4}}=\bar{z}_{1}\left(1-\varepsilon \frac{z_{2}}{z_{1}}\right) \quad(\omega \in D H-A), \quad \text { dual - hyperbolic conjugation } \\
& |\omega|^{\dagger_{5}}=z_{2}-\varepsilon z_{1}, \quad \text { anti }- \text { dual conjugation }
\end{aligned}
$$

where "- " denotes the standard complex conjugation and the zero divisors of $D H$ is defined by the set $A$ [6]. Namely, $D H-A$ is a multiplicative group. The dual hyperbolic numbers form a commutative ring with 0 characteristic. Unlike quaternions, the multiplication of dual-hyperbolic numbers with generalized Fibonacci and Lucas number has a commutative ring structure. However, multiplication of dual-hyperbolic numbers with generalized Fibonacci and Lucas number constitutes two-dimensional Complex Clifford and 4-dimensional Real Clifford algebra structure.

\section{Properties of Dual-Hyperbolic numbers with generalized Fibonacci and Lucas coefficients}

The dual-hyperbolic Fibonacci and Lucas numbers are defined as

$$
D H F_{n}=F_{n}+F_{n+1} j+F_{n+2} \varepsilon+F_{n+3} j \varepsilon
$$

and

$$
D H L_{n}=L_{n}+L_{n+1} j+L_{n+2} \varepsilon+L_{n+3} j \varepsilon
$$

respectively. Here, $F_{n}$ and $L_{n}$ are the $n$-th generalized Fibonacci number and Lucas numbers respectively and $\varepsilon$ denotes dual unit $\left(\varepsilon^{2}=0, \varepsilon \neq 0\right), j$ denotes imaginary unit $\left(j^{2}=1\right), j \varepsilon$ denotes imaginary-dual unit $\left(j \varepsilon^{2}=0\right)$. After these numbers have been defined in the article [1], some identities regarding the modules, conjugates have been obtained for dual-hyperbolic Fibonacci and Lucas numbers. Then, negadual-hyperbolic Fibonacci, negadual-hyperbolic Lucas, d'Ocagne's, Cassini, Catalan identities and the correspondence of Binet formula have been given for these numbers. Now, Let's define the dual-hyperbolic number system with generalized Fibonacci and Lucas coefficients by considering the study [1].

Definition 3.1. $H_{n}$ is called as $n$-th Fibonacci number which have either $H_{n}=H_{n-1}+H_{n-2}, n \geq 3$ or $H_{n}=(p-q) F_{n}+q F_{n+1}$ the recurrence relations and depending on the initial values such that

$$
H_{1}=p, H_{2}=p+q, H_{3}=2 q+3 p, \ldots \quad(p, q \in Z) .
$$

Then, the sets of generalized Fibonacci and Lucas sequences are defined

$$
D H X=\left\{D H X_{n}=R_{n}+\varepsilon R_{n}^{*}=\left(H_{n}+j H_{n+1}\right)+\varepsilon\left(H_{n+2}+j H_{n+3}\right) \mid H_{n} \text { Generalized Fibonacci Number }\right\}
$$

and

$$
D H Y=\left\{D H Y_{n}=P_{n}+\varepsilon P_{n}^{*}=\left(V_{n}+j V_{n+1}\right)+\varepsilon\left(V_{n+2}+j V_{n+3}\right) \mid V_{n} \text { Generalized Lucas Number }\right\}
$$

where $\varepsilon\left(\varepsilon^{2}=0, \varepsilon \neq 0\right), j\left(j^{2}=1\right)$ and $i \varepsilon\left((j \varepsilon)^{2}=0\right)$, denote dual unit, imaginary unit and dual-imaginary unit, respectively. So the base elements of dual-hyperbolic numbers with generalized Fibonacci and Lucas coefficients are $(1, j, \varepsilon, j \varepsilon)$. Multiplication scheme of these base elements are given in Table 1. 


\begin{tabular}{|c||c|c|c|c|}
\hline$\times$ & 1 & $j$ & $\varepsilon$ & $j \varepsilon$ \\
\hline \hline 1 & 1 & $j$ & $\varepsilon$ & $j \varepsilon$ \\
\hline$j$ & $j$ & 1 & $j \varepsilon$ & $\varepsilon$ \\
\hline$\varepsilon$ & $\varepsilon$ & $j \varepsilon$ & 0 & 0 \\
\hline$j \varepsilon$ & $j \varepsilon$ & $\varepsilon$ & 0 & 0 \\
\hline
\end{tabular}

Table 1: Multiplication scheme of dual-hyperbolic units

If two dual-hyperbolic numbers with generalized Fibonacci coefficients are $D H X_{n}^{1}=R_{n}+R_{n}^{*} \varepsilon=H_{n}+H_{n+1} j+H_{n+2} \varepsilon+H_{n+3} j \varepsilon$ and $D H X_{n}^{2}=K_{n}+K_{n}^{*} \varepsilon=G_{n}+G_{n+1} j+G_{n+2} \varepsilon+G_{n+3} j \varepsilon$ then the addition, subtraction and multiplication operations of these numbers are defined as

$$
\begin{aligned}
D H X_{n}^{1} \pm D H X_{n}^{2} & =\left(R_{n}+R_{n}^{*} \varepsilon\right) \pm\left(K_{n}+K_{n}^{*} \varepsilon\right) \\
& =\left(H_{n}+H_{n+1} j+H_{n+2} \varepsilon+H_{n+3} j \varepsilon\right) \pm\left(G_{n}+G_{n+1} j+G_{n+2} \varepsilon+G_{n+3} j \varepsilon\right) \\
& =\left(H_{n} \pm G_{n}\right)+\left(H_{n+1} \pm G_{n+1}\right) j+\left(H_{n+2} \pm G_{n+2}\right) \varepsilon+\left(H_{n+3} \pm G_{n+3}\right) j \varepsilon
\end{aligned}
$$

and

$$
\begin{aligned}
D H X_{n}^{1} \times D H X_{n}^{2} & =\left(R_{n}+R_{n}^{*} \varepsilon\right) \times\left(K_{n}+K_{n}^{*} \varepsilon\right) \\
& =\left(H_{n}+H_{n+1} j+H_{n+2} \varepsilon+H_{n+3} j \varepsilon\right) \times\left(G_{n}+G_{n+1} j+G_{n+2} \varepsilon+G_{n+3} j \varepsilon\right) \\
& +\left(H_{n} G_{n}+H_{n+1} G_{n+1}\right)+\left(H_{n} G_{n+1}+H_{n+1} G_{n}\right) j \\
& +\left(H_{n} G_{n+2}+H_{n+1} G_{n+3}+H_{n+3} G_{n+1}+H_{n+2} G_{n}\right) \varepsilon \\
& +\left(H_{n} G_{n+3}+H_{n+1} G_{n+2}+H_{n+2} G_{n+1}+H_{n+3} G_{n}\right) j \varepsilon
\end{aligned}
$$

respectively. Also, any dual-hyperbolic number with generalized Fibonacci coefficient can be expressed as follows

$$
D H X_{n}=R_{n}+R_{n}^{*} \varepsilon=\left(H_{n}+H_{n+1} j\right)+\left(H_{n+2}+H_{n+3} j\right) \varepsilon .
$$

This yields five different conjugates. Thus, these five different conjugates can be defined as follows

$$
\begin{array}{ll}
D H X_{n}^{\dagger 1}=\left(H_{n}-H_{n+1} j\right)+\left(H_{n+2}-H_{n+3} j\right) \varepsilon, & \text { hyperbolic conjugation } \\
D H X_{n}^{\dagger 2}=\left(H_{n}+H_{n+1} j\right)-\left(H_{n+2}+H_{n+3} j\right) \varepsilon, & \text { dual conjugation } \\
D H X_{n}^{\dagger_{3}}=\left(H_{n}-H_{n+1} j\right)-\left(H_{n+2}-H_{n+3} j\right) \varepsilon, & \text { coupled conjugation } \\
D H X_{n}^{\dagger_{4}}=\left(H_{n}-H_{n+1} j\right) \times\left(1-\frac{H_{n+2}+H_{n+3} j}{H_{n}+H_{n+1} j} \varepsilon\right), & \text { dual-hyperbolic conjugation } \\
D H X_{n}^{\dagger 5}=\left(H_{n+2}+H_{n+3} j\right)-\left(H_{n}+H_{n+1} j\right) \varepsilon, &
\end{array}
$$

Five different norms can be given for dual-hyperbolic numbers with generalized Fibonacci coefficients thanks to the definition of conjugates.

Definition 3.2. Let $D H X_{n}$ be a dual-hyperbolic number with generalized Fibonacci coefficient. In this case, $j$-th modulus of DHX $X_{n}$ are denoted by $\left|D H X_{n}\right|_{\dagger_{i}}^{2},(j=1,2,3,4,5)$ and are given as follows

$$
\begin{aligned}
& \left|D H X_{n}\right|_{\dagger_{1}}^{2}=D H H_{n} \times D H H_{n}^{\dagger 1} \\
& \left|D H X_{n}\right|_{\dagger_{2}}^{\eta_{2}}=D H H_{n} \times D H H_{n}^{\dagger 2} \\
& \left|D H X_{n}\right|_{\dagger_{3}}^{2}=D H H_{n} \times D H H_{n}^{\dagger 3} \\
& \left|D H X_{n}\right|_{\dagger_{4}}^{2}=D H H_{n} \times D H H_{n}^{\dagger 4} \\
& \left|D H X_{n}\right|_{\dagger_{5}}^{2}=D H H_{n} \times D H H_{n}^{\dagger 5}
\end{aligned}
$$

Proposition 3.3. Let $\mathrm{DH} X_{n}$ be a dual-hyperbolic number with generalized Fibonacci coefficient. Then, the following identities are satisfied:

$$
\begin{gathered}
D H X_{n}+D H X_{n}^{\dagger 1}=2\left(H_{n}+H_{n+2} \varepsilon\right) \\
D H X_{n} \times D H X_{n}^{\dagger 1}=\left(H_{n}^{2}-H_{n+1}^{2}\right)+2 \varepsilon\left(H_{n} H_{n+2}-H_{n+1} H_{n+3}\right) \\
D H X_{n}+D H X_{n}^{\dagger 2}=2\left(H_{n}+H_{n+1} j\right) \\
D H X_{n} \times D H X_{n}^{\dagger 2}=\left[(2 p-q) H_{2 n+1}-e F_{2 n+1}\right]+2 H_{n} H_{n+1} j
\end{gathered}
$$




$$
\begin{gathered}
D H X_{n}+D H X_{n}^{\dagger 3}=2\left(H_{n}+H_{n+3} j \varepsilon\right) \\
D H X_{n} \times D H X_{n}^{\dagger}=\left(H_{n}^{2}-H_{n+1}^{2}\right)+j \varepsilon\left[2 e(-1)^{n+1}\right] \\
D H X_{n}+D H X_{n}^{\dagger 4}=2 H_{n}+\varepsilon \frac{2 H_{n+1}}{H_{n}^{2}-H_{n+1}^{2}}\left[\left(H_{n+3} H_{n}-H_{n+1} H_{n+2}\right)+j\left(H_{n+2} H_{n}-H_{n+1} H_{n+3}\right)\right] \\
D H X_{n} \times D H X_{n}^{\dagger 4}=H_{n}^{2}-H_{n+1}^{2} \\
D H X_{n}+D H X_{n}^{\dagger}=\left(H_{n}+H_{n+2}\right)+\left(H_{n+1}+H_{n+3}\right) j+H_{n+1} \varepsilon+H_{n+2} j \varepsilon \\
D H X_{n} \times D H X_{n}^{\dagger 5}=\left(H_{n} H_{n+2}+H_{n+1} H_{n+3}\right)+j\left(H_{n} H_{n+3}+H_{n+1} H_{n+2}\right) \\
+\varepsilon\left(H_{n+2}^{2}+H_{n+3}^{2}-H_{n+1}^{2}-H_{n}^{2}\right)+2 j \varepsilon\left(H_{n+3} H_{n+2}-H_{n+1} H_{n}\right)
\end{gathered}
$$

Proof. (3.9): Using equations (3.1), (3.3) and (3.4), we obtain

$$
D H X_{n}+D H X_{n}^{\dagger 1}=2\left(H_{n}+H_{n+2} \varepsilon\right) .
$$

Here, If the values $p=1, q=0$ are specially taken in the generalized Fibonacci number $H_{n}$, then it is concluded that $D H X_{n}+D H X_{n}^{\dagger 1}=$ $2\left(F_{n}+F_{n+2} \varepsilon\right)$.

(3.10): Considering equations (3.2), (3.3) and (3.4), the result is found by

$$
\begin{aligned}
D H X_{n} \times D H X_{n}^{\dagger 1} & =\left(H_{n}^{2}+H_{n+1}^{2}\right)+2\left(H_{n} H_{n+2}+H_{n+1} H_{n+3}\right) \varepsilon \\
& =\left(H_{n}^{2}-H_{n+1}^{2}\right)+2 \varepsilon\left(H_{n} H_{n+2}-H_{n+1} H_{n+3}\right) .
\end{aligned}
$$

Here, If the values $p=1, q=0$ are specially taken in the generalized Fibonacci number $H_{n}$, then it is concluded that $D H X_{n} \times D H X_{n}^{\dagger 1}=$ $F_{2 n+1}+2 F_{2 n+3} \varepsilon$.

(3.11): From the equations (3.1), (3.3) and (3.5), we can reach the following identity

$$
D H X_{n}+D H X_{n}^{\dagger 2}=2\left(H_{n}+H_{n+1} j\right) .
$$

Here, If the values $p=1, q=0$ are specially taken in the generalized Fibonacci number $H_{n}, D H X_{n}+D H X_{n}^{\dagger 1}=2\left(F_{n}+F_{n+2} j\right)$.

(3.12): Using the equations (3.2), (3.3), (3.5), using the identity $H_{n-1}^{2}+H_{n}^{2}=(2 p-q) H_{2 n-1}-e F_{2 n-1}$ (see.ref. [2]) and simplifying we have

$$
D H X_{n} \times D H X_{n}^{\dagger 2}=\left[(2 p-q) H_{2 n+1}-e F_{2 n+1}\right]+2 H_{n} H_{n+1} j
$$

Here, If the values $p=1, q=0$ are specially taken in the generalized Fibonacci number $H_{n}$, then it is concluded that $D H X_{n} \times D H X_{n}^{\dagger 2}=$ $F_{2 n+1}+2 F_{n} F_{n+1} j$.

(3.13): We can write the following equation by using the equations (3.1), (3.3) and (3.6)

$$
D H X_{n}+D H X_{n}^{\dagger 3}=2\left(H_{n}+H_{n+3} j \varepsilon\right) .
$$

Here, If the values $p=1, q=0$ are specially taken in the generalized Fibonacci number $H_{n}$, then it is concluded that $D H X_{n}+D H X_{n}^{\dagger 3}=$ $2\left(F_{n}+F_{n+3} j \varepsilon\right)$.

(3.14): From equations (3.2), (3.3) and (3.6), we have

$$
D H X_{n} \times D H X_{n}^{\dagger}=\left(H_{n}^{2}-H_{n+1}^{2}\right)+j \varepsilon\left[2 e(-1)^{n+1}\right] .
$$

While we are obtaining the above equation, the identity $H_{n} H_{n+r+1}-H_{n-s} H_{n+r+s+1}=(-1)^{n+s} e F_{s} F_{r+s+1}$ has been used [2]. Here, If the values $p=1, q=0$ are specially taken in the generalized Fibonacci number $H_{n}$, then it is concluded that $D H X_{n} \times D H X_{n}^{\dagger 3}=$ $-F_{n-1} F_{n+2}-2(-1)^{n} j \varepsilon$.

(3.15): If we take into account the equations (3.1), (3.3) and (3.7), then the following identity can be easily seen

$$
D H X_{n}+D H X_{n}^{\dagger 4}=2 H_{n}+\varepsilon \frac{2 H_{n+1}}{H_{n}^{2}-H_{n+1}^{2}}\left[\left(H_{n+3} H_{n}-H_{n+1} H_{n+2}\right)+j\left(H_{n+2} H_{n}-H_{n+1} H_{n+3}\right)\right] .
$$


Here, If the values $p=1, q=0$ are specially taken in the generalized Fibonacci number $H_{n}$, then it is concluded that

$$
D H X_{n}+D H X_{n}^{\dagger}=2 F_{n}+\varepsilon \frac{2 F_{n+1}}{F_{n}^{2}-F_{n+1}^{2}}\left[\left(F_{n+3} F_{n}-F_{n+1} F_{n+2}\right)+j\left(F_{n+2} F_{n}-F_{n+1} F_{n+3}\right)\right] .
$$

(3.16): By making the necessary operations with the help of the equations (3.2), (3.3), (3.7) and rearranging the last equation, the following identity can be given

$$
D H X_{n} \times D H X_{n}^{\dagger}=H_{n}^{2}-H_{n+1}^{2}
$$

Here, If the values $p=1, q=0$ are specially taken in the generalized Fibonacci number $H_{n}$, then it is concluded that $D H X_{n} \times D H X_{n}^{\dagger 4}=$ $F_{n}^{2}-F_{n+1}^{2}$.

(3.17): Considering the equations (3.1), (3.3) and (3.8), we have

$$
D H X_{n}+D H X_{n}^{\dagger 5}=\left(H_{n}+H_{n+2}\right)+\left(H_{n+1}+H_{n+3}\right) j+H_{n+1} \varepsilon+H_{n+2} j \varepsilon
$$

Here, If the values $p=1, q=0$ are specially taken in the generalized Fibonacci number $H_{n}$, then it is concluded that $D H X_{n}+D H X_{n}^{\dagger 5}=$ $F_{n}+F_{n+2}+\left(F_{n+1}+F_{n+3}\right) J+F_{n+1} \varepsilon+F_{n+2} j \varepsilon$.

(3.18): If we use equations (3.2), (3.3), (3.8) and make the necessary calculations, then the rearranged equation yields

$$
\begin{aligned}
D H X_{n} \times D H X_{n}^{\dagger 5} & =\left(H_{n} H_{n+2}+H_{n+1} H_{n+3}\right)+j\left(H_{n} H_{n+3}+H_{n+1} H_{n+2}\right) \\
& +\varepsilon\left(H_{n+2}^{2}+H_{n+3}^{2}-H_{n+1}^{2}-H_{n}^{2}\right)+2 j \varepsilon\left(H_{n+3} H_{n+2}-H_{n+1} H_{n}\right) .
\end{aligned}
$$

Here, If the values $p=1, q=0$ are specially taken in the generalized Fibonacci number $H_{n}$, then it is concluded that

$$
\begin{aligned}
D H X_{n} \times D H X_{n}^{\dagger 5} & =\left(F_{n} F_{n+2}+F_{n+1} F_{n+3}\right)+j\left(F_{n} F_{n+3}+F_{n+1} F_{n+2}\right) \\
& +\varepsilon\left(F_{n+2}^{2}+F_{n+3}^{2}-F_{n+1}^{2}-F_{n}^{2}\right)+2 j \varepsilon\left(F_{n+3} F_{n+2}-F_{n+1} F_{n}\right) .
\end{aligned}
$$

Theorem 3.4. Let $D H X_{n}$ and $D H X_{n-1}$ be two dual-hyperbolic numbers with generalized coefficients. There exist the following identities for these numbers and their conjugates:

i) $\left(D H X_{n} \times D H X_{n}^{\dagger 1}\right)+\left(D H X_{n-1} \times D H X_{n-1}^{\dagger 1}\right)=-\left[(2 p-q) H_{2 n}-e F_{2 n}\right]+2 \varepsilon\left(-H_{n+1}^{2}\right)$

ii) $D H X_{n}^{2}=2 H_{n} D H X_{n}-\left(D H X_{n} \times D H X_{n}^{\dagger 1}\right)+2 \varepsilon\left(H_{n+2}^{2}-H_{n+1} H_{n+3}\right)+2 j \varepsilon\left(H_{n+1} H_{n+2}\right)$

iii) $D H X_{n}^{2}+D H X_{n-1}^{2}=2(2 p-q) D H X_{2 n-1}-D H X_{n} \times D H X_{n}^{\dagger 1}-D H X_{n-1} \times D H X_{n-1}^{\dagger 1}+(2 p-q)\left(2 H_{2 n+3} \varepsilon+2 H_{2 n+2} j \varepsilon\right)$

iii) $-e\left(2 F_{2 n-1}+2 F_{2 n} j+2\left(F_{2 n+3}+F_{2 n+1}\right) \varepsilon+4 F_{2 n+2} i \varepsilon\right)-2 H_{n+1}^{2} \varepsilon$

iv) $D H Y_{n} \times D H X_{n}^{\dagger 1}-D H Y_{n}^{\dagger 1} \times D H X_{n}=(-1)^{n}\left[\left(4 p^{2}-8 p q+8 q^{2}\right)+\left(-6 p^{2}\right) j \varepsilon\right]$.

Proof. i) By using identity $H_{n-1}^{2}+H_{n}^{2}=(2 p-q) H_{2 n-1}-e F_{2 n-1}$ [2] and considering the equations (3.2), (3.3) and the above equations which have been defined by Horadam, the proof can be seen easily.

ii) Considering the equation (3.1), the proof can be easily seen.

iii) From the identity $H_{n} H_{m}+H_{n+1} H_{m+1}=(2 p-q) H_{m+n+1}-e F_{m+n+1}$ [3] and equation (3.1), the proof is completed.

iv) Using the equation (3.1) and the identity $L_{n} F_{m}=F_{m+n}+(-1)^{m} F_{m-n}$ [2], the desired result is obtained. Also, the equations given in Proposition 2.2. in the article [1] are specially obtained by giving values $p=1, q=0$ in the equations we have found above.

i) $\left(D H X_{n} \times D H X_{n}^{\dagger 1}\right)+\left(D H X_{n-1} \times D H X_{n-1}^{\dagger 1}\right)=-F_{2 n}+2 \varepsilon\left(-F_{n+1}^{2}\right)$

ii) $D H X_{n}^{2}=2 H_{n} D H X_{n}-\left(D H X_{n} \times D H X_{n}^{\dagger 1}\right)+2 \varepsilon\left(F_{n+2}^{2}-F_{n+1} F_{n+3}\right)+2 j \varepsilon\left(F_{n+1} F_{n+2}\right)$

iii) $D H X_{n}^{2}+D H X_{n-1}^{2}=4 D H X_{2 n-1}-D H X_{n} \times D H X_{n}^{\dagger 1}-D H X_{n-1} \times D H X_{n-1}^{\dagger 1}+\left[-2 F_{2 n-1}+2\left(F_{2 n+3}-F_{2 n+1}\right) \varepsilon-2 F_{2 n} j\right]-2 F_{n+1}^{2} \varepsilon$

iv) $D H Y_{n} \times D H X_{n}^{\dagger 1}-D H Y_{n}^{\dagger 1} \times D H X_{n}=(-1)^{n}[4+(-6) j \varepsilon]$

Theorem 3.5. Let $\mathrm{DH} X_{n}$ be a dual-hyperbolic number with generalized coefficient. Then, the following identities are valid:

1) $D H X_{n}+D H X_{n+1}=D H X_{n+2}$

2) $\left(D H X_{n}\right)^{2}=2\left(H_{n} D H X_{n}\right)+2\left(H_{n+1} D H X_{n+1}\right)-\left[\left(H_{n}^{2}+H_{n+1}^{2}\right)+2\left(H_{n+1} H_{n+2}\right) j+2\left(H_{n+1} H_{n+3}\right) j \varepsilon\right]$

3) $-D H X_{n}+D H X_{n+1} j+D H X_{n+2} \varepsilon-D H X_{n+3} j \varepsilon=H_{\mathrm{n}+1}$ 
4)

$\left(D H X_{n} \times D H X_{m}\right)+\left(D H X_{n+1} \times D H X_{m+1}\right)=(2 p-q)\left[\left(H_{m+n+1}+H_{m+n+3}\right)+2 H_{m+n+2 j}+2\left(H_{m+n+3}+H_{m+n+5}\right) \varepsilon+4 H_{m+n+4} i \varepsilon\right]$ $-e\left[\left(F_{m+n+1}+F_{m+n+3}\right)+2 F_{m+n+2} j+2\left(F_{m+n+3}+F_{m+n+5}\right) \varepsilon+4 F_{m+n+4} j \varepsilon\right]$

5)

$D H X_{n}^{2}+D H X_{n-1}^{2}=\left[(2 p-q)\left(H_{2 n+1}+H_{2 n-1}\right)-e\left(F_{2 n+1}+F_{2 n-1}\right)\right]+2 j\left[(2 p-q) H_{2 n}-e F_{2 n}\right]$

$$
+2 \varepsilon\left[(2 p-q)\left(H_{2 n+3}+H_{2 n+1}\right)-e\left(F_{2 n+3}+F_{2 n+1}\right)\right]+4 j \varepsilon\left[(2 p-q) H_{2 n+2}-e F_{2 n+2}\right]
$$

Proof. 1) Let $D H X_{n}$ and $D H X_{n+1}$ be two dual-hyperbolic numbers with generalized coefficients. In this case, taking into account that the equation (3.1), we get

$$
D H X_{n}+D H X_{n+1}=H_{n+2}+H_{n+3} j+H_{n+4} \varepsilon+H_{n+5} j \varepsilon=D H X_{n+2} .
$$

Here, if the values $p=1$ and $q=0$ are specially taken in the generalized Fibonacci number $H_{n}$, then it is concluded that $D H X_{n}+D H X_{n+1}=$ $\mathrm{DHF}_{n+2}$.

2) Let $\mathrm{DHX} X_{n}$ be dual-hyperbolic numbers with generalized coefficients. If the equation (3.2) is used, then the following equality is obtained

$$
\begin{aligned}
D H X_{n}^{2} & =\left[\left(H_{n}+H_{n+1} j\right)+\left(H_{n+2}+H_{n+3} j\right) \varepsilon\right] \times\left[\left(H_{n}+H_{n+1} j\right)+\left(H_{n+2}+H_{n+3} j\right) \varepsilon\right] \\
& =2\left(H_{n} D H X_{n}\right)+2\left(H_{n+1} D H X_{n+1}\right)-\left[\left(H_{n}^{2}+H_{n+1}^{2}\right)+2\left(H_{n+1} H_{n+2}\right) j+2\left(H_{n+1} H_{n+3}\right) j \varepsilon\right] .
\end{aligned}
$$

Here, if the values $p=1$ and $q=0$ are specially taken in the generalized Fibonacci number $H_{n}$, then $\left(D H X_{n}\right)^{2}=2\left(F_{n} D H F_{n}\right)+$ $2\left(F_{n+1} D H F_{n+1}\right)-\left[\left(F_{n}^{2}+F_{n+1}^{2}\right)+2\left(F_{n+1} F_{n+2}\right) j+2\left(F_{n+1} F_{n+3}\right) j \varepsilon\right]$ is found.

3) By considering the equation (3.1) and doing some algebraic calculations, we obtain

$$
\begin{aligned}
-D H X_{n}+D H X_{n+1} j+D H X_{n+2} \varepsilon-D H X_{n+3} j \varepsilon & =-\left[\left(H_{n}+H_{n+1} j\right)+\left(H_{n+2}+H_{n+3} j\right) \varepsilon\right] \\
& +\left[\left(H_{n+1}+H_{n+2} j\right)+\left(H_{n+3}+H_{n+4} j\right) \varepsilon\right] j \\
& +\left[\left(H_{n+2}+H_{n+3} j\right)+\left(H_{n+4}+H_{n+5} j\right) \varepsilon\right] \varepsilon \\
& -\left[\left(H_{n+3}+H_{n+4} j\right)+\left(H_{n+5}+H_{n+6} j\right) \varepsilon\right] i \varepsilon \\
& =H_{\mathrm{n}+1} .
\end{aligned}
$$

Here, if the values $p=1$ and $q=0$ are specially taken in the generalized Fibonacci number $H_{n}$,

$$
-D H X_{n}+D H X_{n+1} j+D H X_{n+2} \varepsilon-D H X_{n+3} j \varepsilon=F_{\mathrm{n}+1}
$$

is found.

4) Follows from the identity $H_{n} H_{m}+H_{n+1} H_{m+1}=(2 p-q) H_{m+n+1}-e F_{m+n+1}$ (see ref. [3]) and using the equation (3.2), we achieve that

$$
\begin{aligned}
\left(D H X_{n} \times D H X_{m}\right)+\left(D H X_{n+1} \times D H X_{m+1}\right) & =\left[\left(H_{n}+H_{n+1} j\right)+\left(H_{n+2}+H_{n+3} j\right) \varepsilon\right] \times\left[\left(H_{m}+H_{m+1} j\right)+\left(H_{m+2}+H_{m+3} j\right) \varepsilon\right] \\
& +\left[\left(H_{n+1}+H_{n+2} j\right)+\left(H_{n+3}+H_{n+4} j\right) \varepsilon\right] \times\left[\left(H_{m+1}+H_{m+2} j\right)+\left(H_{m+3}+H_{m+4} j\right) \varepsilon\right] \\
& =(2 p-q)\left[\begin{array}{c}
\left(H_{m+n+1}+H_{m+n+3}\right)+2 H_{m+n+2} j+2\left(H_{m+n+3}+H_{m+n+5}\right) \varepsilon \\
+4 H_{m+n+4} i \varepsilon
\end{array}\right] \\
& -e\left[\left(F_{m+n+1}+F_{m+n+3}\right)+2 F_{m+n+2} j+2\left(F_{m+n+3}+F_{m+n+5}\right) \varepsilon+4 F_{m+n+4} j \varepsilon\right]
\end{aligned}
$$

Here, If the values $p=1$ and $q=0$ are specially taken in the generalized Fibonacci number $H_{n}$

$$
\left(D H X_{n} \times D H X_{m}\right)+\left(D H X_{n+1} \times D H X_{m+1}\right)=\left(F_{m+n+1}+F_{m+n+3}\right)+2 F_{m+n+2} j+2\left(F_{m+n+3}+F_{m+n+5}\right) \varepsilon+4 F_{m+n+4} j \varepsilon
$$

5) Considering the identity $H_{n} H_{m}+H_{n+1} H_{m+1}=(2 p-q) H_{m+n+1}-e F_{m+n+1}$ (see ref. [3]) and the equation (3.2), we reach the result. Here, If the values $p=1$ and $q=0$ are specially taken in the generalized Fibonacci number $D H X_{n}{ }^{2}+D H X_{n-1}^{2}=\left(F_{2 n+1}+F_{2 n-1}\right)+$ $2 F_{2 n} j+2\left(F_{2 n+3}+F_{2 n+1}\right) \varepsilon+4 F_{2 n+2} j \varepsilon$.

Theorem 3.6. Let $\mathrm{DH} X_{n}$ and $\mathrm{DH} \mathrm{L}_{n}$ be dual-hyperbolic Fibonacci and dual-hyperbolic Lucas numbers with generalized Fibonacci and Lucas coefficients, respectively. For $n \geq 0$, there exist the following relationships between these numbers:

1) $D H X_{n+1}+D H X_{n-1}=p D H L_{n}+q D H L_{n}$

2) $D H X_{n+2}-D H X_{n-2}=p D H L_{n}+q D H L_{n}$

Proof. Equations 1) and 2) are found by taking the identity $H_{n+1}+H_{n-1}=p L_{n}+q L_{n-1}$ (see ref. [4]) and using the recurrence relation $H_{n}=(p-q) F_{n}+q F_{n+1}$, respectively.

$$
\begin{aligned}
D H X_{n+1}+D H X_{n-1} & =\left(H_{n+1}+H_{n+2} j+H_{n+3} \varepsilon+H_{n+4} j \varepsilon\right)+\left(H_{n-1}+H_{n} j+H_{n+1} \varepsilon+H_{n+3} j \varepsilon\right) \\
& =\left(H_{n+1}+H_{n-1}\right)+\left(H_{n+2}+H_{n}\right) j+\left(H_{n+3}+H_{n+1}\right) \varepsilon+\left(H_{n+4}+H_{n+3}\right) j \varepsilon \\
& =\left(p L_{n}+q L_{n-1}\right)+\left(p L_{n-1}+q L_{n}\right) j+\left(p L_{n+2}+q L_{n+1}\right) \varepsilon+\left(q L_{n+3}+q L_{n+2}\right) j \varepsilon \\
& =p D H L_{n}+q D H L_{n}
\end{aligned}
$$




$$
\begin{aligned}
D H X_{n+2}-D H X_{n-2} & =\left(H_{n+2}+H_{n+3} j+H_{n+4} \varepsilon+H_{n+5} j \varepsilon\right)-\left(H_{n-2}+H_{n-1} j+H_{n} \varepsilon+H_{n+1} j \varepsilon\right) \\
& =\left(H_{n+2}-H_{n-2}\right)+\left(H_{n+3}-H_{n-1}\right) j+\left(H_{n+4}-H_{n}\right) \varepsilon+\left(H_{n+5}-H_{n+1}\right) j \varepsilon \\
& =\left(p L_{n}+q L_{n-1}\right)+\left(p L_{n-1}+q L_{n}\right) j+\left(p L_{n+2}+q L_{n+1}\right) \varepsilon+\left(q L_{n+3}+q L_{n+2}\right) j \varepsilon \\
& =p D H L_{n}+q D H L_{n}
\end{aligned}
$$

Here, if the values $p=1, q=0$ are specially taken in the generalized Fibonacci number $H_{n}$, then the desired results are obtained.

Theorem 3.7. The sums of the dual-hyperbolic numbers with generalized Fibonacci coefficients satisfy the following relations:

1) $\sum_{s=1}^{n} D H X_{s}=D H X_{n+2}-D H X_{2}$

2) $\sum_{s=0}^{p} D H X_{s+n}+D H X_{x+1}=D H X_{n+p+2}$

3) $\sum_{s=1}^{n} D H X_{2 s-1}=D H X_{2 n}-D H X_{0}$

4) $\sum_{s=1}^{n} D H X_{2 s}=D H X_{2 n+1}-D H X_{1}$

Proof. Using the identity $\sum_{t=a}^{n} H_{t}=H_{n+2}-H_{a+1}$ (see ref. [4]), the proof can be seen easily as follows

1) $\sum_{s=1}^{n} D H X_{s}=\sum_{s=1}^{n} H_{s}+j \sum_{s=1}^{n} H_{s+1}+\varepsilon \sum_{s=1}^{n} H_{s+2}+j \varepsilon \sum_{s=1}^{n} H_{s+3}=D H X_{n+2}-D H X_{2}$

2) $\sum_{s=0}^{p} D H X_{n+s}+D H X_{n+1}=\sum_{s=1}^{n} H_{n+s}+H_{n+1}+j \sum_{s=1}^{n} H_{n+s+1}+H_{n+2}+\varepsilon \sum_{s=1}^{n} H_{n+s+2}+H_{n+3}+j \varepsilon \sum_{s=1}^{n} H_{n+s+3}+H_{n+4}=D H X_{n+p+2}$

3) $\sum_{s=1}^{n} D H X_{2 s-1}=\sum_{s=1}^{n} H_{2 s-1}+j \sum_{s=1}^{n} H_{2 s}+\varepsilon \sum_{s=1}^{n} H_{2 s+1}+j \varepsilon \sum_{s=1}^{n} H_{2 s+2}=D H X_{2 n}-D H X_{0}$

4) $\sum_{s=1}^{n} D H X_{2 s}=\sum_{s=1}^{n} H_{2 s}+j \sum_{s=1}^{n} H_{2 s+1}+\varepsilon \sum_{s=1}^{n} H_{2 s+2}+j \varepsilon \sum_{s=1}^{n} H_{2 s+3}=D H X_{2 n+1}-D H X_{1}$

Also, if we consider the values $p=1, q=0$ in the generalized Fibonacci number $H_{n}$, then the above equations becomes as follows:

1) $\sum_{s=1}^{n} D H X_{s}=D H F_{n+2}-D H F_{2}$

2) $\sum_{s=0}^{p} D H X_{s+n}+D H X_{x+1}=D H F_{n+p+2}$

3) $\sum_{s=1}^{n} D H X_{2 s-1}=D H F_{2 n}-D H F_{0}$

4) $\sum_{s=1}^{n} D H X_{2 s}=D H F_{2 n+1}-D H F_{1}$

Now, let's find correspondence of the Binet formula for the dual-hyperbolic Fibonacci numbers which helps to find golden ratio.

Theorem 3.8. Let $D H X_{n}$ be dual-hyperbolic number with generalized Fibonacci coefficient. For $m, n \geq 1$, the Binet formula for this number is given by

$$
D H X_{n}=\frac{\bar{\alpha} \alpha^{n}-\bar{\beta} \beta^{n}}{\alpha-\beta}
$$

where $\alpha=\frac{1+\sqrt{5}}{2}, \beta=\frac{1-\sqrt{5}}{2}$ and the coefficients $\bar{\alpha}, \bar{\beta}$ are as follows

$$
\bar{\alpha}=(p-q \beta)+[p(1-\beta)+q] j+[p(2-\beta)+q(1-\beta)] \varepsilon+[p(3-2 \beta)+q(2-\beta)] j \varepsilon
$$

and

$$
\bar{\beta}=(p-q \alpha)+[p(1-\alpha)+q] j+[p(2-\alpha)+q(1-\alpha)] \varepsilon+[p(3-2 \alpha)+q(2-\alpha)] j \varepsilon .
$$

Proof. If $t_{1}$ and $t_{2}$ denote the roots of characteristic equation $t^{2}-t-1=0$ associated to the recurrence relation $D H X_{n}+D H X_{n+1}=D H X_{n+2}$. Then, these roots can be found as $\alpha=t_{1}=\frac{1+\sqrt{5}}{2}$ and $\beta=t_{2}=\frac{1-\sqrt{5}}{2}$. Note that, $\alpha+\beta=1, \alpha \cdot \beta=-1$ and $\alpha-\beta=\sqrt{5}$. Therefore, the general term of the dual-hyperbolic number sequence with generalized Fibonacci coefficients may be expressed in the form:

$$
D H X_{n}=A \alpha^{n}+B \beta^{n}
$$

for some coefficients $A$ and $B$. For $n=0$ and $n=1$, the following equalities can be written

$$
D H X_{0}=(q, p, p+q, 2 p+q)
$$

and

$$
D H X_{1}=(p, p+q, 2 p+q, 3 p+2 q) .
$$


Also, if we give to $n$ the values $n=0$ and $n=1$, we get

$$
D H X_{0}=A+B
$$

and

$$
D H X_{1}=\alpha A+\beta B
$$

Then, solving this system of linear equations, we have

$$
A=\frac{D H X_{1}-\beta D H X_{0}}{\alpha-\beta} \quad \text { and } \quad B=\frac{\alpha D H X_{0}-D H X_{1}}{\alpha-\beta}
$$

where some coefficients $\bar{\alpha}$ and $\bar{\beta}$ are

$$
\bar{\alpha}=(p-q \beta)+[p(1-\beta)+q] j+[p(2-\beta)+q(1-\beta)] \varepsilon+[p(3-2 \beta)+q(2-\beta)] j \varepsilon
$$

and

$$
\bar{\beta}=(p-q \alpha)+[p(1-\alpha)+q] j+[p(2-\alpha)+q(1-\alpha)] \varepsilon+[p(3-2 \alpha)+q(2-\alpha)] j \varepsilon
$$

Theorem 3.9. The generating function for dual-hyperbolic number with generalized coefficients is

$$
g(x)=\frac{1}{1-x-x^{2}} \sum_{s=0}^{3}\left(D H X_{s}+D H X_{s-1} x\right) e_{s} .
$$

Proof. Assuming that the generating function for dual-hyperbolic number with generalized coefficients becomes

$$
g(x)=\sum_{n=0}^{\infty} P_{n} x^{n} .
$$

such that

$$
P_{n}=\left(D H X_{n}, D H X_{n+1}, D H X_{n+2}, D H X_{n+3}\right) .
$$

Multiplying the generating function by $x$ and $x^{2}$, the following equalities can be written

$$
\begin{aligned}
& x g(x)=P_{0} x+P_{1} x^{2}+\ldots+P_{n-1} x^{n}+\ldots \\
& x^{2} g(x)=P_{0} x^{2}+P_{1} x^{3}+\ldots+P_{n-2} x^{n}+\ldots
\end{aligned}
$$

After some algebraic calculations, we obtain

$$
g(x)=\frac{1}{1-x-x^{2}} \sum_{s=0}^{3}\left(P_{0}+\left(P_{1}-P_{0}\right) x\right) .
$$

This completes the proof.

Now, let's write the Binet formula in terms of the generating function which has been obtained in Theorem 3.9.

Theorem 3.10. Binet formula for the dual-hyperbolic numbers with generalized Fibonacci coefficients is

$$
P_{n}=P_{1} H_{n}+P_{0} H_{n-1}
$$

Proof. Let's we take the relation

$$
P_{n}=A \alpha^{n}+B \beta^{n}
$$

Putting $n=0$ and $n=1$ in the above equation, $A$ and $B$ are obtained by

$$
A=\frac{P_{1}-\beta P_{0}}{\alpha-\beta}, \quad B=\frac{\alpha P_{0}-P_{1}}{\alpha-\beta}
$$

In this case, $P_{n}$ can be rewritten as

$$
P_{n}=\frac{1}{\alpha-\beta}\left[\left(P_{1}-\beta P_{0}\right) \alpha^{n}+\left(\alpha P_{0}-P_{1}\right) \beta^{n}\right] .
$$

When the equalities of $P_{0}$ and $P_{1}$ is written in Theorem 3.9 and is arranged, $P_{n}$ is found as

$$
P_{n}=\left(\frac{\alpha^{n}-\beta^{n}}{\alpha-\beta}\right) \sum_{s=0}^{3} D H X_{s+1} e_{s}+\left(\frac{\alpha^{n-1}-\beta^{n-1}}{\alpha-\beta}\right) \sum_{s=0}^{3} D H X_{s} e_{s}
$$

Finally

$$
P_{n}=P_{1} H_{n}+P_{0} H_{n-1}
$$

is obtained. 
Let us express the Catalan identity which is one of the most known identities of Fibonacci numbers.

Theorem 3.11. (Catalan's Identity)

For $n \geq r$, the relation

$$
D H X_{n}^{2}-D H X_{n+r} \times D H X_{n-r}=(-1)^{n-r} \mu F_{r}^{2}[j+3 j \varepsilon]
$$

is verified.

Proof. Squaring $D H X_{n}$, multiplying $D H X_{n+r}$ and $D H X_{n-r}$ and noting that $H_{m+k} H_{n-k}-H_{m} H_{n}=(-1)^{n-k+1} \mu F_{k} F_{m+k-n}$ [7], the following equalities are obtained

$$
D H X_{n}^{2}=H_{n}^{2}+H_{n+1}^{2}+2 H_{n} H_{n+1} j+2\left(H_{n} H_{n+2}+H_{n+1} H_{n+3}\right) \varepsilon+2\left(H_{n+1} H_{n+2}+H_{n} H_{n+3}\right) j \varepsilon
$$

and

$$
\begin{aligned}
\operatorname{DHX}_{n+r} \times D H X_{n-r} & =H_{n+r} H_{n-r}+H_{n+r+1} H_{n-r+1}+\left(H_{n+r+1} H_{n-r}+H_{n+r} H_{n-r+1}\right) j \\
& +\left(H_{n+r} H_{n-r+2}+H_{n+r+1} H_{n-r+3}+H_{n-r+2} H_{n-r}+H_{n-r+3} H_{n-r+1}\right) \varepsilon \\
& +\left(H_{n+r+1} H_{n-r+2}+H_{n+r} H_{n-r+3}+H_{n+r+3} H_{n-r}+H_{n+r+2} H_{n-r+1}\right) j \varepsilon .
\end{aligned}
$$

Adding the above equations gives us the proof. Writting $p=1$ and $q=0$ in the Catalan identity for dual-hyperbolic numbers with generalized coefficients, Catalan identity for dual-hyperbolic numbers with Fibonacci coefficients is found. Namely

$$
D H X_{n}^{2}-D H X_{n+r} \times D H X_{n-r}=(-1)^{n-r} F_{r}^{2}(j+3 j \varepsilon) .
$$

Let's give Cassini identity for generalized dual-hyperbolic numbers as a special case of Catalan identity.

Theorem 3.12. (Cassini's Identity)

Let $\mathrm{DHX}_{n}$ be the dual-hyperbolic number with generalized Fibonacci coefficients. For $n \geq 1$, we have

$$
D H X_{n}^{2}-\left(D H X_{n+1} \times D H X_{n-1}\right)=(-1)^{n-1} \mu(j+3 j \varepsilon) .
$$

Proof. For $r=1$, we see that the identity in Theorem 3.11 becomes the desired identity.Putting $p=1$ and $q=0$ in the above identity, we get

$$
D H X_{n}^{2}-\left(D H X_{n+1} \times D H X_{n-1}\right)=(-1)^{n-1}(j+3 j \varepsilon) .
$$

This identity is Cassini formula for dual-hyperbolic numbers.

Theorem 3.13. (Honsberger Identity)

For $n, m \geq 0$, the Honsberger identity for the dual-hyperbolic number with generalized coefficient $D H X_{n}$ is given by

$$
\begin{aligned}
\left(D H X_{k-1} \times D H X_{n}\right)+\left(D H X_{k} \times D H X_{n+1}\right) & =\left[(2 p-q)\left(H_{k+n}+H_{k+n+2}\right)-e\left(F_{k+n}+F_{k+n+2}\right)\right] \\
& +2 j\left[(2 p-q) H_{k+n+1}-e F_{k+n+1}\right] \\
& +2 \varepsilon\left[(2 p-q)\left(H_{k+n+2}+H_{k+n+4}\right)-e\left(F_{k+n+2}+F_{k+n+4}\right)\right] \\
& +4 j \varepsilon\left[(2 p-q) H_{k+n+3}-e F_{k+n+3}\right] .
\end{aligned}
$$

Proof. If we take into consider the equations (3.1), (3.2) and use the identity $H_{n} H_{m}+H_{n+1} H_{m+1}=(2 p-q) H_{m+n+1}-e F_{m+n+1}$ (see ref. [3]), we complete the proof. If the values $p=1, q=0$ are specially taken in the generalized Fibonacci number $H_{n}$, then the following identity is found

$$
\begin{aligned}
\left(D H X_{k-1} \times D H X_{n}\right)+\left(D H X_{k} \times D H X_{n+1}\right) & =\left(F_{k+n}+F_{k+n+2}\right)+2 F_{k+n+1} j \\
& +2\left(F_{k+n+2}+F_{k+n+4}\right) \varepsilon+4 F_{k+n+3} j \varepsilon .
\end{aligned}
$$

Theorem 3.14. (Tagiuri Identity)

Let $\mathrm{DHX}_{n}$ be the dual-hyperbolic number with generalized Fibonacci coefficients. For $m n, m \geq 1$, Tagiuri's identity is as follows:

$$
\left(D H X_{m+k} \times D H X_{n-k}\right)-\left(D H X_{m} \times D H X_{n}\right)=(-1)^{n-k-1} \mu F_{k} F_{m+k-n}(j+3 j \varepsilon) .
$$

Proof. The proof can be easily seen by using the identity $H_{m+k} H_{n-k}-H_{m} H_{n}=(-1)^{n-k+1} \mu F_{k} F_{m+k-n}$ [7] and equations (3.1) and (3.2). If the values $p=1, q=0$ are specially taken in the generalized Fibonacci number $H_{n}$, then it is concluded that

$$
\left(D H X_{m+k} \times D H X_{n-k}\right)-\left(D H X_{m} \times D H X_{n}\right)=(-1)^{n-k-1} F_{k} F_{m+k-n}(j+3 j \varepsilon) .
$$

Theorem 3.15. (d'Ocagne's Identity)

Let $\mathrm{DHX}_{n}$ be the dual-hyperbolic number with generalized Fibonacci coefficients. For $m>n, m \in N$ and $n \in Z$, we have

$$
\left(D H X_{m+k} \times D H X_{n-k}\right)-\left(D H X_{m} \times D H X_{n}\right)=\mu F_{m-n}(-1)^{n}(j+3 j \varepsilon) .
$$

Proof. Using identity $H_{m+k} H_{n-k}-H_{m} H_{n}=(-1)^{n-k+1} \mu F_{k} F_{m+k-n}$ [7] and the equations (3.1) and (3.2), the proof is completed. If the values $p=1, q=0$ are specially taken in the generalized Fibonacci number $H_{n}$, then the following identity is found

$$
\left(D H X_{m+k} \times D H X_{n-k}\right)-\left(D H X_{m} \times D H X_{n}\right)=F_{m-n}(-1)^{n}(j+3 j \varepsilon) .
$$




\section{Conclusion}

Our main aim in this study was to generalize the study which was done on dual-hyperbolic numbers. It was seen that, some theorems were obtained as a result of this generalization and they corresponded to the theorems in the article [1] for $p=1, q=0$. Also, the generating function was obtained and the Binet formula was given with the help of the generating function. Unlike the identities which was given in the article [1], Honsberger and Tagiuri identities were proved. At the same time, special cases of these identities were discussed. Because of the fact that generalized Fibonacci and Lucas coefficient dual-hyperbolic number system have commutative algebra structure, five different conjugates can be defined. As a result, in addition to the identities related to the conjugates which we obtained in Proposition 3.4 , the following identities are given.

i)

$$
\begin{aligned}
& \left(D H X_{n} \times D H X_{n}^{\dagger 2}\right)+\left(D H X_{n-1} \times D H X_{n-1}^{\dagger 2}\right)=(2 p-q)\left[H_{2 n-1}+H_{2 n+2}\right]-e\left[F_{2 n-1}+F_{2 n+2}\right]+2 j H_{n} H_{n-1} \\
& \left(D H X_{n} \times D H X_{n}^{\dagger_{3}}\right)+\left(D H X_{n-1} \times D H X_{n-1}^{\dagger_{3}}\right)=-(1+2 j)\left[(2 p-q) H_{2 n}-e F_{2 n}\right] \\
& \left(D H X_{n} \times D H X_{n}^{\dagger 4}\right)+\left(D H X_{n-1} \times D H X_{n-1}^{\dagger 4}\right)=-\left[(2 p-q) H_{2 n}-e F_{2 n}\right] \\
& \left(D H X_{n} \times D H X_{n}^{\dagger 5}\right)+\left(D H X_{n-1} \times D H X_{n-1}^{\dagger 5}\right)=\left[(2 p-q)\left(H_{2 n+3}+H_{2 n+1}\right)-e\left(F_{2 n+3}+F_{2 n+1}\right)\right] \\
& +2 j\left[(2 p-q) H_{2 n+2}-e F_{2 n+2}\right] \\
& +\varepsilon\left[(2 p-q)\left(2 H_{2 n}+H_{2 n+5}\right)-e\left(2 F_{2 n+3}+F_{2 n+5}\right)\right] \\
& +2 j \varepsilon\left[(2 p-q)\left(H_{2 n+2}+H_{2 n}\right)-e\left(F_{2 n+2}+F_{2 n}\right)\right]
\end{aligned}
$$

ii)

$$
\begin{aligned}
D H X_{n}^{2} & =2 H_{n} D H X_{n}-D H X_{n} D H X_{n}^{\dagger 2}+2 H_{n+1}\left(H_{n+1}+H_{n} j+H_{n+2} \varepsilon+H_{n+2} j \varepsilon\right) \\
D H X_{n}^{2} & =2 H_{n} D H X_{n}-D H X_{n} D H X_{n}^{\dagger 3}+2\left(H_{n+2} H_{n+1} \varepsilon+H_{n} H_{n+3} j \varepsilon\right) \\
D H X_{n}^{2} & =2 H_{n} D H X_{n}-D H X_{n} D H X_{n}^{\dagger 4}+2 H_{n+2}\left(H_{n} \varepsilon+H_{n+1} j \varepsilon\right) \\
D H X_{n}^{2} & =2 H_{n} D H X_{n}-D H X_{n} D H X_{n}^{\dagger 5}+\left(H_{n+2} H_{n-1}+H_{n+2} H_{n}+H_{n+1} H_{n+3}\right) \\
& +\left(H_{n+3} H_{n}+H_{n+1} H_{n+2}\right) j+\left(2 H_{n+2} H_{n}+H_{n+2}^{2}+H_{n+3}^{2}-H_{n+1}^{2}-H_{n}^{2}\right) \varepsilon \\
& +2\left(H_{n+2} H_{n+1}+H_{n+2} H_{n+3}-H_{n+1} H_{n}\right) \mathrm{j} \varepsilon
\end{aligned}
$$

iii)

$$
\begin{aligned}
D H X_{n}^{2}+D H X_{n-1}^{2} & =2(2 p-q) D H X_{2 n-1}-D H X_{n} D H X_{n}^{\dagger 2}-D H X_{n-1} D H X_{n-1}^{\dagger_{2}} \\
& +(2 p-q)\left(2 H_{2 n+1}+2 H_{2 n+3} \varepsilon+2 H_{2 n+2} j \varepsilon\right) \\
& -e\left(2 F_{2 n-1}+2 F_{2 n+1}+F_{2 n} j+2\left(F_{2 n+3}+F_{2 n+1}\right) \varepsilon+4 F_{2 n+2} j \varepsilon\right)+2\left(H_{n} H_{n-1}\right) j \\
D H X_{n}^{2}+D H X_{n-1}^{2} & =2(2 p-q) D H X_{2 n-1}-D H X_{n} D H X_{n}^{\dagger 3}-D H X_{n-1} D H X_{n-1}^{\dagger 3} \\
& +(2 p-q)\left(-2 H_{2 n} j+2 H_{2 n+3} \varepsilon+2 H_{2 n+2} j \varepsilon\right) \\
& -e\left(2 F_{2 n-1}+2\left(F_{2 n+3}+F_{2 n+1}\right) \varepsilon+4 F_{2 n+2} j \varepsilon\right) \\
& =2(2 p-q) D H X_{2 n-1}-D H X_{n} D H X_{n}^{\dagger 4}-D H X_{n-1} D H X_{n-1}^{\dagger 4} \\
& +2(2 p-q)\left(H_{2 n+3} \varepsilon+H_{2 n+2} j \varepsilon\right) \\
& -2 e\left(F_{2 n-1}+F_{2 n} j+\left(F_{2 n+3}+F_{2 n+1}\right) \varepsilon+2 F_{2 n+2} j \varepsilon\right) \\
& =2(2 p-q) D H X_{2 n-1}-D H X_{n} D H X_{n}^{\dagger 5}-D H X_{n-1} D H X_{n-1}^{\dagger}+D H X_{n-1}^{\dagger_{5}} \\
& +(2 p-q)\left[H_{2 n+4}+2 H_{2 n+2} j+\left(2 H_{2 n+3}+2 H_{2 n}+H_{2 n+5}\right) \varepsilon+2\left(2 H_{2 n+2}+H_{2 n}\right) j \varepsilon\right] \\
& -e\left[F_{2 n+3}+2 F_{2 n+1}+F_{2 n-1}+2\left(F_{n}+F_{2 n+2}\right)+\left(4 F_{2 n+3}+F_{2 n+5}\right) \varepsilon+\left(6 F_{2 n+2}+F_{2 n}\right) j \varepsilon\right]
\end{aligned}
$$

iv)

$$
\begin{aligned}
D H Y_{n} \times D H X_{n}^{\dagger_{2}}-D H Y_{n}^{\dagger_{2}} \times D H X_{n} & =4(-1)^{n}\left[2 p^{2} \varepsilon+p^{2} j \varepsilon\right] \\
D H Y_{n} \times D H X_{n}^{\dagger_{3}}-D H Y_{n}^{\dagger_{3}} \times D H X_{n} & =4(-1)^{n}\left[\left(p^{2}-2 p q+2 q^{2}\right) j-2 p^{2} \varepsilon\right] \\
D H Y_{n} \times D H X_{n}^{\dagger_{4}}-D H Y_{n}^{\dagger_{4}} \times D H X_{n} & =\left[\begin{array}{l}
\left.4(-1)^{n-1}\left(p^{2}+2 p q-2 q^{2}\right)\right] j \\
+
\end{array}\right. \\
& +\frac{8(-1)^{n} p^{2}}{\left(V_{n}+V_{n+1} i\right)\left(H_{n}+H_{n+1} i\right)}\left[\begin{array}{l}
-p^{2} F_{2 n+1}+p q\left(-2 F_{2 n+2}-F_{2 n}\right) \\
+q^{2}\left(F_{2 n+1}+F_{2 n}\right)
\end{array}\right] j \varepsilon \\
D H Y_{n} \times D H X_{n}^{\dagger_{5}}-D H Y_{n}^{\dagger_{5}} \times D H X_{n} & =2(-1)^{n-1} p^{2} j
\end{aligned}
$$

The proofs of these identities are easily seen by following the similar ways in the proof of Theorem 3.4. Finally, the special values $p=1$ and $q=0$ provide the above equations. 


\section{References}

[1] A. Cihan, A. Z. Azak, M. A. Güngör, M. Tosun, Investigation of Dual-hyperbolic Fibonacci, Dual-hyperbolic Lucas Numbers and their properties. An. Stiiinţ. Univ. "Ovidius" Constanța Ser. Mat., 27(1), 35-48(2019)

[2] A. F. Horadam, A generalized Fibonacci sequence, Amer. Math. Monthly, 68 (1961), 455-459.

[3] A. L. Iakini, Generalized quaternions of higher order, Fibonacci Quart., 15 (1977), 343-346.

[4] S. Yüce, F. Aydın Torunbalc1, A new aspect of dual Fibonacci quaternions, Adv. Appl. Clifford Algebr., 26 (2015), 873-884.

[5] F. Torunbalcı Aydın, Hyperbolic Fibonacci sequence, Univers. J. Math. Appl., 2(2) (2019), 59-64.

[6] F. Messelmi, Dual-complex numbers and their Holomorphic functions, https://hal.archives-ouvertes.fr/hal-01114178, (2015).

[7] T. Koshy, Fibonacci and Lucas Numbers with Applications, Wiley and Sons Publication, New York, 2001. 